\title{
Antiviral Nanodelivery Systems: Current Trends in Acyclovir Administration
}

\author{
Haniza Hassan, ${ }^{1}$ Siti Khadijah Adam, ${ }^{1}$ Fauziah Othman, ${ }^{1}$ \\ Ahmad Fuad Shamsuddin, ${ }^{2,3}$ and Rusliza Basir ${ }^{1}$ \\ ${ }^{1}$ Department of Human Anatomy, Faculty of Medicine and Health Sciences, Universiti Putra Malaysia (UPM), \\ 43400 Serdang, Selangor, Malaysia \\ ${ }^{2}$ Centre for Drug Delivery Research, Faculty of Pharmacy, Universiti Kebangsaan Malaysia, Jalan Raja Muda Abdul Aziz, \\ 50300 Kuala Lumpur, Malaysia \\ ${ }^{3}$ Faculty of Pharmacy \& Health Sciences, Universiti Kuala Lumpur Royal College of Medicine Perak, No. 3, Jalan Greentown, \\ 30450 Ipoh, Perak, Malaysia
}

Correspondence should be addressed to Ahmad Fuad Shamsuddin; drafsna@gmail.com and Rusliza Basir; rusliza@upm.edu.my

Received 4 April 2016; Revised 19 July 2016; Accepted 25 July 2016

Academic Editor: Changyang Gong

Copyright (C) 2016 Haniza Hassan et al. This is an open access article distributed under the Creative Commons Attribution License, which permits unrestricted use, distribution, and reproduction in any medium, provided the original work is properly cited.

\begin{abstract}
Poor bioavailability of acyclovir in the treatment of viral infections remains one of the major drug delivery concerns of pharmaceutical manufacturers and researchers. Nanoparticulate systems have been exploited with the aim of improving the current pharmacological limitations of acyclovir administration. In fact, nanoparticles do offer many advantages, especially in terms of their physicochemical stability and sustained-release properties. Besides, they are made of biocompatible materials, which are nontoxic to cells. Acyclovir has been a focus since the last decade as one of the low bioavailability drug models loaded in various types of newly synthesized drug delivery vehicles. In this review, compositions and formulations of nanosized acyclovir particles, as well as their stability and pharmacokinetic profile, are discussed in further detail.
\end{abstract}

\section{Introduction}

Acyclovir is an antiviral drug that is primarily used for the treatment of HSV (herpes simplex virus) infection as well as infections due to varicella zoster and herpes zoster. It has higher activity towards both HSV-1 and HSV-2 as compared to the latter viral infections. Acyclovir remains the drug of choice for prophylaxis and treatment of HSV infection and is available in numerous forms such as tablet, suspension, intravenous injection, and ophthalmic ointment (Table 1).

Acyclovir is a guanosine analogue with a functional aliphatic group that is present on the side chain. It obstructs the virus replication process by competitively inhibiting the viral DNA formation. This is achieved through selective binding of HSV-thymidine kinase. Acyclovir is then converted by the viral thymidine kinase to acycloguanosine monophosphate (acyclo-GMP) followed by phosphorylation by the cellular kinase into an active triphosphate form; acycloguanosine triphosphate (acyclo-GTP). The acyclo-GTP, which has greater affinity for the viral DNA polymerase as compared to cellular polymerase, functions as a substrate that binds with and is incorporated into viral DNA. Eventually, this results in premature chain termination as acyclo-GTP resembles the nucleotides but without the hydroxyl group in the $3^{\prime}$ position [1-3]. The absence of this hydroxyl group means that further nucleotides cannot be added to the strand for elongation and, hence, the incomplete DNA strand is terminated (Figure 1). As a consequence, the replication rate is slowed down and further provides ample time for the immune response to intervene.

Acyclovir is poorly soluble in water contributing to only $20 \%$ of the total free drug in the body. It is also rapidly cleared from the system where the half-life of the drug is reported to be three hours. Unchanged acyclovir and its metabolite, 9-carboxy-methoxymethylguanine, can be found in the urine as kidneys are the major organs for the drugs' elimination [4]. By virtue of this, acyclovir does not have sufficient bioavailability, accounting for only up to $30 \%$ in 
TABLE 1: Route of administration of acyclovir and the disadvantages.

\begin{tabular}{lll}
\hline Route of administration & Dose & Disadvantages \\
\hline Oral & $1000 \mathrm{mg}$ per day (tablet and suspension) & $\begin{array}{l}\text { Low oral bioavailability, high-dose related side effects, that } \\
\text { is, nausea, headache, and diarrhoea [8, 15] }\end{array}$ \\
Intravenous injection & $100 \mathrm{mg} / \mathrm{kg}$ & $\begin{array}{l}\text { Nephrotoxicity, renal failure, and thrombophlebitis [21, 28] } \\
\text { Difficulty in penetrating epidermal layer, rapid clearance }\end{array}$ \\
Topical/transdermal & $5 \% \mathrm{w} / \mathrm{w}$ topical ointment, 5 times daily & [29] \\
Ocular & $3 \% \mathrm{w} / \mathrm{w}$ ophthalmic ointment, 5 times a day & Poor ocular bioavailability [30] \\
\hline
\end{tabular}

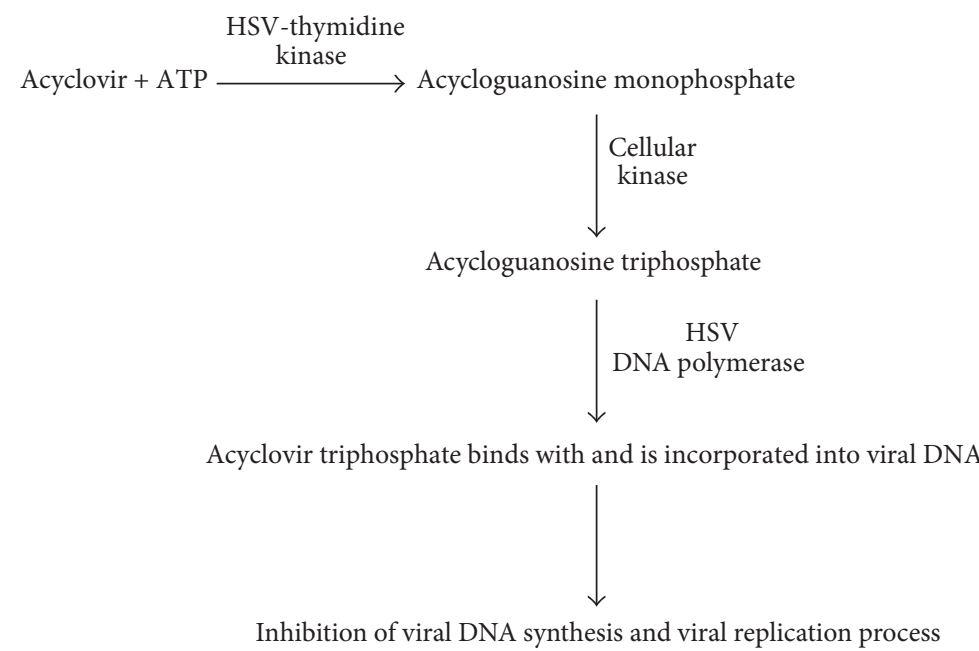

FIGURE 1: Schematic mechanism of action of acyclovir in inhibiting viral DNA synthesis.

immunocompetent patient with no previous history of renal problem or complication [5-7]. Nonetheless, this percentage varies from one individual to another and it is subjected to the individual pharmacokinetic profile of patients. Poor bioavailability of acyclovir has led to ineffective therapy. A simplistic approach to overcome this is by prescribing acyclovir in high oral doses, that is, $200 \mathrm{mg}$ tablet, five times daily for ten consecutive days or even higher doses of $400 \mathrm{mg}$, if required. Higher doses are prescribed by physicians to obtain higher serum concentration, hence, achieving its desired therapeutic effects [8-10]. Nonetheless, the use of higher drug doses may lead to unwarranted adverse effects.

New or enhanced techniques and formulations are needed to improve the efficacy of the current antiviral therapy using acyclovir. Less frequent dosing, lower dose of acyclovir used, and sustained-release mechanisms are approaches utilised to improve therapy and reduce the possibility of getting side effects related to high dose of drug consumed. This can very much improve patient compliance to antiviral therapy using acyclovir. Research in the nanoparticulate system as drug carriers has received intense and worldwide attention. This novel system is seen to be the next step in improving antiviral therapy. Nanoparticles have been selected to deliver drugs effectively with minimal to no toxicity effects [11-13]. Usage of nanoparticles in medicine has brought forward more options in developing future drug, as well as improving the currently available formulations, which are inundated with pharmacological limitations.
Nanosized drug delivery vehicle has been expected to fulfil the main pharmacokinetic objectives, that is, improving acyclovir's solubility and, hence, absorption and oral bioavailability. Moreover, it is believed that nanosized acyclovir may potentially improve the drug's dissolution profile thus overcoming its pharmacokinetic drawback. For these reasons, various types of nanosized drug delivery vehicles have been studied, formulated, and prepared by using different methods to encapsulate and deliver acyclovir via different routes of administration. This drug delivery system includes synthetic and natural products, which are safe for consumers. These products are made of biologically inert and physiologically compatible materials. Besides, most nanocarriers are formulated to be biodegradable which is helpful in avoiding toxicity. Acyclovir nanocarriers are discussed in detail with respect to their route of administration.

\section{Oral Delivery}

The purpose of high doses of oral acyclovir (typically a $200 \mathrm{mg}$ tablet, taken 5 times a day for 10 days) prescribed to patients is to achieve the desired acyclovir concentration circulated in plasma, thus attaining significant pharmacological effect of acyclovir. Notably, oral delivery is the most preferred route of administration. However, patient compliance is compromised if acyclovir oral tablets are required to be taken five times daily. On top of that, the patient is exposed to side effects related to high doses of acyclovir 
administration. Some of the common side effects of orally delivered acyclovir are nausea, vomiting, diarrhoea, and headache [8]. In order to obtain therapeutic effect of as well as enhancing compliance and minimising its dose related side effects, various techniques and methods for delivery of acyclovir have been developed.

A lipid nanoemulsion system has been designed and introduced to entrap acyclovir. This emulsion system is made up of liquid lipids, which are dispersed in water and stabilized by emulsifier and/or surfactant that coat the nanosized lipid droplets. Emulsifiers or surfactants are used to reduce the interfacial tension of lipid droplets with the aqueous phase (water). Surfactant and emuslifiers are added in the formulation to avoid droplets collision in order to maintain the desired droplet size [14]. Although the emulsifying technique is quite an antiquated drug preparation technique, the modern technology has adapted this method to a level where it stands together with other newer drug delivery forms. Moreover, an improvement in drug solubility will definitely have a positive impact on the drug bioavailability.

Nanoemulsion systems for oral acyclovir delivery have been shown to improve acyclovir oral bioavailability in rats when compared with the commercially available plain drug either in tablet form or in solutions. More importantly, the particles produced were within the nanometric size, ranging from $20 \mathrm{~nm}$ to $40 \mathrm{~nm}$ with good drug entrapment and loading capacity $[15,16]$. In addition, liquid nanoemulsion developed showed longer blood circulation time when compared with plain drug solution. In contrast, it was reported that the selfmicroemulsifying drug delivery system, which was made up of $30 \%$ glycerol, $9 \%$ sunflower oil, and $60 \%$ tween 60 , did not exhibit a sustained-release profile. Also, the half-life $\left(t_{1 / 2}\right)$ was reported to be about 2.5 to 3 hours which was similar to the pure drug solution [15]. These studies showed that types and ratio of the oil to surfactant (optimized using pseudoternary phase diagram) used in formulating the nanoemulsion played a major role in determining the sustained-release characteristic of the nanoemulsions constructed.

More recently, an in vitro drug release study of acyclovirloaded Eudragi RLPO nanoparticles showed sustained drug release property for over a period of 24 hours. However, the use of Eudragi RLPO and a stabilizer, Pluronic F68, might not be a good combination for formulation of oral drug delivery vehicle, due to the fact that range of particle size produced was large, between 82 and $532 \mathrm{~nm}$. The drug entrapment efficiency was reported to be moderate at about $53 \%-79 \%$ [17]. Thus, the composition and the ratio of both surfactant and polymer used in the formulation directly influenced the drug entrapment efficiency and its particle sizes, as well as the polydispersity index. In addition, large sizes and high polydispersity index particles are indicators of instability of a formulation and are benchmarked to estimate the nanoparticle's shelf-life storage condition.

A stability test is one of the most crucial steps in developing drug carrier. Usually, the stability test of a newly formulated drug is an indicator of the product performances over time and an important criterion for future drug commercialization. The shelf-life stability of the nanoemulsion formulation is an interesting area to study as some of the emulsions prepared had been said to face instability problems that include separation and sedimentation of the prepared formulation. Storage at room temperature for more than 6 months would cause the prepared emulsion containing acyclovir to change in its globule size significantly. Drug burst/leakage has also been reported despite its good entrapment efficiency, oral bioavailability, and prolonged release characteristic [18]. However, the system was stable if it was kept in controlled temperature $\left(4^{\circ} \mathrm{C}\right)[18,19]$. Thus, further investigation into the compositions, as well as the types of lipid used, would give insightful and clearer perspective of the oral drug delivery system proposed.

\section{Systemic Blood Delivery (Intravenous (IV) Injections)}

Numerous attempts have been made by scientists and researchers to develop acyclovir nanocarrier for IV injection. In general, bolus IV injection is only given to patients if very high doses of acyclovir are required, that is, $100 \mathrm{mg} / \mathrm{kg}$, and/or when serious cases of HSV infection are encountered such as herpes simplex encephalitis [20].

However, delivery of acyclovir via IV injection would cause thrombophlebitis at the injection area. Also, high doses of acyclovir could cause deposition of acyclovir crystals in the kidney, especially if acyclovir is given via bolus IV injection, where the maximum solubility is reached. This event may lead to intratubular renal damage and, hence, renal failure [21-23]. This is one of the common side effects if acyclovir dose is not adjusted or proper hydration is not given [24].

Novel IV stealthy nanoparticles using polylactic coglycolic acid (PLGA) have been developed to address the inherent issue of poor acyclovir bioavailability. The objective of this new system is to increase its mean residence time and plasma half-life. The PLGA nanovehicles have been proven to extend circulation time (longer mean residence time) for acyclovir, that is, up to 48 hours, when compared to its control (plain drug solution). Studies have also shown that sphericalshaped PLGA nanoparticles have relatively good percentage of drug entrapment, where more than $60 \%$ of drug added was entrapped in this carrier system. Acyclovir in PLGA nanocarrier yielded a reasonable particle size ranging from $200 \mathrm{~nm}$ to $300 \mathrm{~nm}[25,26]$.

A study in the use of acyclovir PLGA nanoparticles in rats has revealed a much improved acyclovir biodistribution in which the concentration of acyclovir PLGA nanoparticles was highest in hepatocytes compared to other organs (i.e., kidney and lungs) after an intravenous injection of the drug was given through the tail vein [26]. This is an example of a successful targeted drug delivery with prolonged and continuous drug release at the site-specific organ, most likely contributed by the types of drug carriers that interacted with/bound to the surrounding cell, in this case, hepatocytes. As acyclovir is also a useful therapeutic agent in treating patients with life-threatening infectious liver disease (e.g., hepatitis B), the study provides promising evidence of utilising this delivery system to carry acyclovir into the body.

Self-assembled nanoparticles (SAN) formulated from acyclovir lipid derivative (SGSA) have been claimed to be 
stable during storage at room temperature. SAN maintained its particle size of $83.2 \mathrm{~nm}$ up to one year of storage and no particles aggregation was observed. Further stability tests on prepared SAN which included high speed centrifugation (up to $8000 \mathrm{rpm}$ ) with addition of selected additives have shown that SGSA did not show any significant difference when compared with the control group. However, SGSA was reported to have a short elimination time ( 7 hours) with cells toxicity observed. This is an indication of a shorter residence/circulation time of acyclovir in blood and cytotoxicity of the nanoparticles should be subjected to close scrutiny in future research [27].

Factors such as composition and compatibility of each component used in the formulation and methods utilised in the production process may impact the physical profiles of the resultant product prepared. These factors may affect the size, stability, and pharmacokinetic and pharmacodynamic characteristics of the nanoparticles formed. Enhanced technique and improved formulations to deliver acyclovir via I.V injection continue to attract interest from researchers and further investigations would be worthy of note.

\section{Topical and Transdermal Applications}

Skin lesion and blisters due to infection of HSV can be treated with the application of acyclovir in the form of topical ointment or transdermal cream. Topical and transdermal formulations act as vehicles for antiviral agents besides rendering protective layer to prevent further virus entry and spread from a particular site of infection. These formulations also improve the healing time of cold sores and blisters as the application is localised at the site of infection. However, acyclovir ointment has been reported to have only moderate efficacy depending on the severity of the infection and the clinical condition of patients. Moreover, frequent applications of acyclovir topical cream are required to attain its therapeutic effect [31, 32]. Also, it has been reported that acyclovir cream and ointment have difficulties in penetrating the epidermal layer of the skin [31]. Due to slow absorption of the drug at the site of application, research into new and improved formulations for acyclovir topical ointment has received significant attention.

Additionally, nanoemulsion and to an extent microemulsion-based topical formulations have been developed for treatment of cutaneous herpes infection. The applications of these acyclovir formulations exhibited good skin permeation profile and resulted in HSV-1 suppression at the site of infection $[33,34]$. A study on the effect of microemulsionbased topical formulations of acyclovir for the treatment of cutaneous herpetic infections showed a positive improvement where no herpetic skin lesion was observed after one week of treatment [34]. In this study, 2.5\% transcutol (ethoxydiglycol) microemulsion containing $0.75 \mathrm{mg}$ of acyclovir was applied once daily to female Balb/c mice at 24 hours after infection and compared to its control group. Furthermore, the formulations were found to be stable when kept on shelf at a room temperature of $25^{\circ} \mathrm{C}$ for up to 45 days [34].

Although further investigations are required to closely scrutinize its antiviral effect, a study has confirmed that application of acyclovir emulsion for treatment of skin HSV infection enhanced the healing time due to improvement of its skin permeation profile. Schwarz and colleage have also postulated that acyclovir nanoemulsions formulations using natural sugar surfactant and Solutol HS 15 could be a suitable candidate for topical acyclovir delivery that should be developed as future drug vehicles [33]. These findings suggested that drug carrier should not only possess good physicochemical stability and drug loading capacity but also be made of skin-friendly materials and be capable of penetrating the skin barrier that allows interaction with the surrounding cells ensuring sustained drug release to the targeted area.

Beside emulsions, colloidal dispersion drug delivery system has also been exploited for dermal delivery of acyclovir. A detailed in vitro comparative study between ethosomes and solid lipid nanoparticles (SLNs) as acyclovir drug carriers reported improved drug encapsulation with comparable ability to carry high amount of acyclovir. The ethosomal system utilising phosphatidylcholine showed $94 \%$ drug encapsulation which was $39 \%$ higher than tristearin solid lipid nanoparticle. These systems showed similar size of particles of $257 \mathrm{~nm}$ and $236 \mathrm{~nm}$, respectively. Evaluation study on antiviral activity of both acyclovir formulations in plaque reduction assay of infected Vero cells cultures produced similar results when compared to control (plain acyclovir solution) [35]. Thus, it could be concluded that both formulations did not affect the efficacy of the drug. In addition, data obtained from an in vitro release kinetic study suggested that ethosomes and SLNs have sustained-release characteristic where continuous drug delivery of acyclovir was observed. This positive characteristic should be further exploited.

Typically, drug prepared in a form of gel using in situ gelling system is suitable for topical administration as it offers site-specific and targeted drug delivery. Encapsulated acyclovir in polymer nanoparticles dispersion gelling system such as Pluronic F127 is not subjected to topical and transdermal application only, but also nasal and vaginal deliveries. Ramyadevi and Sandhya reported that regardless of its wide range of particles size distribution (between $68 \mathrm{~nm}$ and $1281 \mathrm{~nm}$ ), the system exhibited reasonable drug loading capacity (between $30 \%$ and $60 \%$ ) with up to 8 hours of sustained-release profile. In fact, both characteristics had fulfilled the main objective of the study: a modified dosage form of acyclovir for delivery to the skin [36]. Further investigations on its in vivo bioavailability would be worthy of additional information for the system developed. Therefore, intense research and work progress in this area are required, that is, the toxicity and the stability of the formulation, in order to improve the current findings for better and promising drug delivery methods and preparations.

\section{Ocular Delivery}

Besides topical ointment, acyclovir has also been targeted for its ocular delivery. Ocular HSV infection occurs when HSV type 1 infects the sensory neurons of the eye. To date, herpetic keratitis is one of the most common infectious eye diseases 
associated with HSV [37]. Prolonged herpes keratitis due to lack of treatment or recurrent infection of HSV at the same area of the cornea would cause dendritic ulcer. Eventually, this event may cause visual impairment or, worse, blindness due to scarring and damage formed in the infected area $[38,39]$.

Acyclovir is not prescribed to patients in the form of eye drops because of its poor ocular bioavailability and therapeutic efficacy due to low solubility in water. On top of that, development of drug for delivery to the eye is very challenging. There are few factors that need to be taken into consideration when designing drug for ocular delivery. Eyes have their own physical and biological protective mechanisms that protect them from foreign substances including drugs. For example, blinking and tears will reduce and/or remove ophthalmic drug solution from the conjunctiva. Therefore, it is quite difficult to get the actual dose of drug to reach the targeted tissue area, hence resulting in low ocular drug absorption [40]. Treatment of ocular HSV in immunocompetent patients is prescription of oral acyclovir (800 mg, daily) and/or continuous application of 3\% acyclovir ophthalmic ointment to the cornea area, five times daily $[30,41]$.

In the last decade, a pioneer study was conducted to investigate the potential of liposome as acyclovir delivery vehicle. Liposome offers great advantages as a colloidal drug carrier; it is made of biodegradable materials and is nontoxic to cells. By virtue of that, nanosized liposomes made of phosphatidylcholine-cholesterol-dimethyl dioctadecyl had been developed to transport acyclovir to the eyes. Characteristics of the liposomes prepared from different procedures and membrane charges were evaluated. Data of the study suggested that only charged membranes (either positively or negatively charged liposomes) could interact with acyclovir. Negatively charged liposomes showed the highest drug entrapment efficiency when compared with neutral and positively charged liposomes, which could be due to greater aqueous phase entrapment within the core where acyclovir was placed. However, only positively charged liposomes showed enhanced ocular acyclovir bioavailability in the aqueous humor. This could be due to corneal permeability adjustment made by liposomes which allows more drug to enter the cornea area [42].

In agreement with previous study, positively charged liposome was also reported to be bound to and coated on the corneal surface and enhanced the absorption of acyclovir in the cornea. Also, longer acyclovir residence time of the positively charged liposomes was observed in the aqueous humor of the rabbits due to corneal adhesion as compared to the commercially available acyclovir ointment $[43,44]$. In summary, a few factors have been discovered to influence the improvement of acyclovir-liposomes ophthalmic delivery, which includes rate of corneal permeation and surface charge of the fabricated liposomes such that it binds to and coats the negatively charged corneal surface.

Besides liposomes, poly(ethylene glycol)-co-cyclic acetal (PECA) and polylactic acid (PLA) nanospheres coated with polyethylene glycol (PEG) with average diameter of $200 \mathrm{~nm}$ could also be a good and suitable drug vehicle for acyclovir.
Acyclovir-loaded PECA and PLA nanospheres have demonstrated sustained-release property with mean residence time of up to 6 hours $[45,46]$. In vivo Draize tests were conducted and findings suggested that nanospheres made from both types of polymer were well tolerated with no eye inflammation observed. Thus, acyclovir-loaded nanospheres were considered safe for ocular use since changes were not seen in the rabbit's eyes.

Acyclovir-loaded PEG coated nanospheres had shown a significant increase of acyclovir concentration in the aqueous humor when compared with acyclovir solution. These could have resulted from higher drug loading capacity and better ocular mucoadhesion of the formulated nanospheres. Good drug loading capacity and high ocular bioavailability are indication of a successful trial of acyclovir encapsulation for its ocular delivery. Data of the studies suggested that drug entrapment efficiency is subjected to environment and preparation conditions (i.e., $\mathrm{pH}$ and temperature) and types and molecular weight of the surfactant and polymer used in the formulation. In addition, the composition, concentration, and ratio of surfactant and polymer used in the formulation might influence the physicochemical properties of lipid nanoparticles and the ocular tolerability [47-49]. Further investigation to test the nanospheres' shelf-life stability will be beneficial for future ocular drug delivery development.

More recently, solid lipid nanoparticles have been introduced to be a carrier system to deliver poorly watersoluble and/or hydrophobic drug. It offers many advantages over the older colloidal drug delivery systems, which include biocompatibility, biodegradability, and being made of safe/nontoxic materials [50,51]. Thus, as a stable drug carrier, solid lipid nanoparticles have been proposed to be a drug vehicle for ophthalmic delivery of acyclovir. Acyclovir has been successfully incorporated into solid lipid nanoparticles made from glyceryl dibehanate, Compritol 888 ATO, using the modified hot-oil-in-water microemulsion technique. The morphology, stability, and other characteristics of the newly synthesized nanoparticles were determined before they were tested in excised bovine cornea. It was reported that the negatively charged nanoparticle displayed poor permeation through cornea although it exhibited sustained-release profile to supply acyclovir for a longer period of time. Hence, in order to overcome the drawback, solid lipid nanoparticles have been physically converted to nanostructured lipid carriers (400-777 $\mathrm{nm}$ in diameter) with good drug loading capacity characteristic [52].

Besides having good drug entrapment efficiency of up to $90 \%$, the nanostructured lipid carrier system also enhanced the acyclovir corneal permeation. This has caused an increment of acyclovir cell uptake and its corneal bioavailability. Apart from that, chitosan-coated nanostructured lipid carrier also improved the antiviral efficacy of acyclovir by 3.5fold after 24-hour exposure in the cornea of albino rabbits when compared with the commercial acyclovir ophthalmic ointment available in the market $[52,53]$. The physicochemical properties and structures of the formulated solid lipid nanoparticles (i.e., where acyclovir is loaded in the system) directly influence the bioavailability of acyclovir and rate of 
corneal permeation as well as its stability. These are among the important measures for a successful development of ophthalmic drug delivery.

\section{Conclusion}

The development of nanosized drug delivery system for antiviral drugs, specifically acyclovir, has shown a very good progress and high potential to become successful for future benefits. The delivery of acyclovir through different routes of administration in different forms of formulations (i.e., tablets, suspension, and ointment) is substantial in treating patients with HSV, where infection can be mild to one individual, but severe to others, depending on their health status. Yet each route of administration has its own disadvantages and side effects. Alternative acyclovir delivery system is indeed a very good research area to explore. The forthcoming commercialization of the end product will be the most exciting part, especially to the pharmaceutical industry. Nonetheless, a few fundamental steps have to be carefully studied before the formulation can be commercialized. Moreover, it is necessary that the selection of delivery vehicle for acyclovir be tested via in vitro and in vivo studies. Its optimized therapeutic efficacy and toxicity data can be attained, as well as its stability test, as a prerequisite for clinical trials. Although further research and studies are required to be performed, the current and up-to-date data from various studies, conducted utilising different methods, technology, and resources, have gained worldwide attention and, thus, have contributed important information in this area of research. In fact, the proposed vehicles have the potential to be commercialized to suit the current needs for antiviral delivery to overcome its pharmacokinetic drawbacks. Research in the nanodelivery system for acyclovir will add on to the latest knowledge and improve our understanding in drug discovery as well as delivery systems. The new technology and system proposed will not only benefit antiviral drug delivery but also benefit other marketed drugs with similar delivery problems.

\section{Competing Interests}

The authors declare that there is no conflict of interests regarding the publication of this paper.

\section{References}

[1] G. B. Elion, "The biochemistry and mechanism of action of acyclovir," Journal of Antimicrobial Chemotherapy, vol. 12, pp. 9-17, 1983.

[2] G. B. Elion, "Acyclovir: discovery, mechanism of action, and selectivity," Journal of Medical Virology, vol. 1, supplement 1, pp. 2-6, 1993.

[3] J. Piret and G. Boivin, "Resistance of herpes simplex viruses to nucleoside analogues: mechanisms, prevalence, and management," Antimicrobial Agents and Chemotherapy, vol. 55, no. 2, pp. 459-472, 2011.

[4] J. P. Smith, S. Weller, B. Johnson, J. Nicotera, J. M. Luther, and D. W. Haas, "Pharmacokinetics of acyclovir and its metabolites in cerebrospinal fluid and systemic circulation after administration of high-dose valacyclovir in subjects with normal and impaired renal function," Antimicrobial Agents and Chemotherapy, vol. 54, no. 3, pp. 1146-1151, 2010.

[5] R. J. Whitley, M. R. Blum, N. Barton, and P. De Miranda, "Pharmacokinetics of acyclovir in humans following intravenous administration. A model for the development of parenteral antivirals," The American Journal of Medicine, vol. 73, no. 1, pp. 165-171, 1982.

[6] J.-M. Poirier, N. Radembino, and P. Jaillon, "Determination of acyclovir in plasma by solid-phase extraction and column liquid chromatography," Therapeutic Drug Monitoring, vol. 21, no. 1, pp. 129-133, 1999.

[7] M. Kubbinga, M. A. Nguyen, P. Staubach, S. Teerenstra, and P. Langguth, "The influence of chitosan on the oral bioavailability of acyclovir-a comparative bioavailability study in humans," Pharmaceutical Research, vol. 32, no. 7, pp. 2241-2249, 2015.

[8] A. Wald, J. Benedetti, G. Davis, M. Remington, C. Winter, and L. Corey, "A randomized, double-blind, comparative trial comparing high- and standard-dose oral acyclovir for firstepisode genital herpes infections," Antimicrobial Agents and Chemotherapy, vol. 38, no. 2, pp. 174-176, 1994.

[9] Y. J. Bryson, M. Dillon, M. Lovett et al., "Treatment of first episodes of genital herpes simplex virus infection with oral acyclovir. A randomized double-blind controlled trial in normal subjects," The New England Journal of Medicine, vol. 308, no. 16, pp. 916-921, 1983.

[10] G. W. Raborn, W. T. McGaw, M. Grace, L. D. Tyrrell, and S. M. Samuels, "Oral acyclovir and herpes labialis: a randomized, double-blind, placebo-controlled study," The Journal of the American Dental Association, vol. 115, no. 1, pp. 38-42, 1987.

[11] H. Shirazi, M. Daneshpour, S. Kashanian, and K. Omidfar, "Synthesis, characterization and in vitro biocompatibility study of $\mathrm{Au} / \mathrm{TMC} / \mathrm{Fe}_{3} \mathrm{O}_{4}$ nanocomposites as a promising, nontoxic system for biomedical applications," Beilstein Journal of Nanotechnology, vol. 6, no. 1, pp. 1677-1689, 2015.

[12] M. B. Chaudhari, P. P. Desai, P. A. Patel, and V. B. Patravale, "Solid lipid nanoparticles of amphotericin B (AmbiOnp): in vitro and in vivo assessment towards safe and effective oral treatment module," Drug Delivery and Translational Research, vol. 6, no. 4, pp. 354-364, 2016.

[13] Y. Oh, N. Lee, H. W. Kang, and J. Oh, "In vitro study on apoptotic cell death by effective magnetic hyperthermia with chitosan-coated $\mathrm{MnFe}_{2} \mathrm{O}_{4}$," Nanotechnology, vol. 27, no. 11, article 115101, 2016.

[14] B. Sapra, P. Thatai, S. Bhandari, J. Sood, M. Jindal, and A. Tiwary, "A critical appraisal of microemulsions for drug delivery-part I," Therapeutic Delivery, vol. 4, no. 12, pp. 1547-1564, 2013.

[15] D. Patel and K. K. Sawant, "Oral bioavailability enhancement of acyclovir by self-microemulsifying drug delivery systems (SMEDDS)," Drug Development and Industrial Pharmacy, vol. 33, no. 12, pp. 1318-1326, 2007.

[16] P. K. Ghosh, R. J. Majithiya, M. L. Umrethia, and R. S. R. Murthy, "Design and development of microemulsion drug delivery system of acyclovir for improvement of oral bioavailability," AAPS PharmSciTech, vol. 7, no. 3, p. 77, 2006.

[17] A. Gandhi, S. Jana, and K. K. Sen, "In-vitro release of acyclovir loaded Eudragit $\mathrm{RLPO}^{\circledR}$ nanoparticles for sustained drug delivery," International Journal of Biological Macromolecules, vol. 67, pp. 478-482, 2014.

[18] S. Paul, A. Kumar, P. Yedurkar, and K. Sawant, "Design and development of multiple emulsion for enhancement of oral 
bioavailability of acyclovir," Drug Development and Industrial Pharmacy, vol. 39, no. 11, pp. 1809-1817, 2013.

[19] B. B. Ghera, F. Perret, Y. Chevalier, and H. Parrot-Lopez, "Novel nanoparticles made from amphiphilic perfluoroalkyl $\alpha$-cyclodextrin derivatives: preparation, characterization and application to the transport of acyclovir," International Journal of Pharmaceutics, vol. 375, no. 1-2, pp. 155-162, 2009.

[20] U. Sili, A. Kaya, A. Mert et al., "Herpes simplex virus encephalitis: clinical manifestations, diagnosis and outcome in 106 adult patients," Journal of Clinical Virology, vol. 60, no. 2, pp. 112-118, 2014.

[21] X. Zhang, Y. Li, H. Zhou et al., "Plasma metabolic profiling analysis of nephrotoxicity induced by acyclovir using metabonomics coupled with multivariate data analysis," Journal of Pharmaceutical and Biomedical Analysis C, vol. 97, pp. 151-156, 2014.

[22] A. Seedat and G. Winnett, "Acyclovir-induced acute renal failure and the importance of an expanding waist line," $B M J$ Case Reports, 2012.

[23] H. Lu, Y.-J. Han, J.-D. Xu, W.-M. Xing, and J. Chen, "Proteomic characterization of acyclovir-induced nephrotoxicity in a mouse model," PLoS ONE, vol. 9, no. 7, Article ID e103185, 2014.

[24] R. Fleischer and M. Johnson, "Acyclovir nephrotoxicity: a case report highlighting the importance of prevention, detection, and treatment of acyclovir-induced nephropathy," Case Reports in Medicine, vol. 2010, Article ID 602783, 3 pages, 2010.

[25] A. O. Kamel, G. A. S. Awad, A. S. Geneidi, and N. D. Mortada, "Preparation of intravenous stealthy acyclovir nanoparticles with increased mean residence time," AAPS PharmSciTech, vol. 10, no. 4, pp. 1427-1436, 2009.

[26] S. Gupta, A. Agarwal, N. K. Gupta, G. Saraogi, H. Agrawal, and G. P. Agrawal, "Galactose decorated PLGA nanoparticles for hepatic delivery of acyclovir," Drug Development and Industrial Pharmacy, vol. 39, no. 12, pp. 1866-1873, 2013.

[27] Y. Jin, L. Tong, P. Ai, M. Li, and X. Hou, "Self-assembled drug delivery systems: 1 . Properties and in vitro/in vivo behavior of acyclovir self-assembled nanoparticles (SAN)," International Journal of Pharmaceutics, vol. 309, no. 1-2, pp. 199-207, 2006.

[28] S. Rao, M. J. Abzug, P. Carosone-Link et al., "Intravenous acyclovir and renal dysfunction in children: A Matched Case Control Study," The Journal of Pediatrics, vol. 166, no. 6, pp. 1462.e4-1468.e4, 2015.

[29] A. B. Nair, "Quantification of uptake and clearance of acyclovir in skin layers," Antiviral Therapy, vol. 21, no. 1, pp. 17-25, 2015.

[30] S. Ghosh, V. Jhanji, E. Lamoureux, H. R. Taylor, and R. B. Vajpayee, "Acyclovir therapy in prevention of recurrent herpetic keratitis following penetrating keratoplasty," American Journal of Ophthalmology, vol. 145, no. 2, pp. 198-202, 2008.

[31] G. E. Parry, P. Dunn, V. P. Shah, and L. K. Pershing, "Acyclovir bioavailability in human skin," The Journal of Investigative Dermatology, vol. 98, no. 6, pp. 856-863, 1992.

[32] S. A. Qureshi, M. Jiang, K. K. Midha, and J. P. Skelly, "In vitro evaluation of percutaneous absorption of an acyclovir product using intact and tape-stripped human skin," Journal of Pharmacy \& Pharmaceutical Sciences, vol. 1, no. 3, pp. 102-107, 1998.

[33] J. C. Schwarz, V. Klang, S. Karall, D. Mahrhauser, G. P. Resch, and C. Valenta, "Optimisation of multiple W/O/W nanoemulsions for dermal delivery of aciclovir," International Journal of Pharmaceutics, vol. 435, no. 1, pp. 69-75, 2012.
[34] G. Shishu, S. Rajan, and Kamalpreet, "Development of novel microemulsion-based topical formulations of acyclovir for the treatment of cutaneous herpetic infections," AAPS PharmSciTech, vol. 10, no. 2, pp. 559-565, 2009.

[35] R. Cortesi, L. Ravani, E. Menegatti, M. Drechsler, and E. Esposito, "Colloidal dispersions for the delivery of acyclovir: A Comparative Study," Indian Journal of Pharmaceutical Sciences, vol. 73, no. 6, pp. 687-693, 2011.

[36] D. Ramyadevi and P. Sandhya, "Dual sustained release delivery system for multiple route therapy of an antiviral drug," Drug Delivery, vol. 21, no. 4, pp. 276-292, 2014.

[37] E. Miserocchi, G. Fogliato, I. Bianchi, F. Bandello, and G. Modorati, "Clinical features of ocular herpetic infection in an Italian referral center," Cornea, vol. 33, no. 6, pp. 565-570, 2014.

[38] S. Burrel, D. Boutolleau, G. Azar et al., "Phenotypic and genotypic characterization of acyclovir-resistant corneal HSV1 isolates from immunocompetent patients with recurrent herpetic keratitis," Journal of Clinical Virology, vol. 58, no. 1, pp. 321-324, 2013.

[39] O. Alekseev, A. H. Tran, and J. Azizkhan-Clifford, "Ex vivo organotypic corneal model of acute epithelial herpes simplex virus type I infection," Journal of Visualized Experiments, no. 69, Article ID e3631, 2012.

[40] C. Bucolo, F. Drago, and S. Salomone, "Ocular drug delivery: a clue from nanotechnology," Frontiers in Pharmacology, vol. 3, article 188, 2012.

[41] D. D. Garcia, Q. Farjo, D. C. Musch, and A. Sugar, "Effect of prophylactic oral acyclovir after penetrating keratoplasty for herpes simplex keratitis," Cornea, vol. 26, no. 8, pp. 930-934, 2007.

[42] M. Fresta, A. M. Panico, C. Bucolo, C. Giannavola, and G. Puglisi, "Characterization and in-vivo ocular absorption of liposome-encapsulated acyclovir," The Journal of Pharmacy and Pharmacology, vol. 51, no. 5, pp. 565-576, 1999.

[43] S. L. Law, K. J. Huang, and C. H. Chiang, "Acyclovir-containing liposomes for potential ocular delivery: corneal penetration and absorption," Journal of Controlled Release, vol. 63, no. 1-2, pp. 135-140, 2000.

[44] P. Chetoni, S. Rossi, S. Burgalassi, D. Monti, S. Mariotti, and M. F. Saettone, "Comparison of liposome-encapsulated acyclovir with acyclovir ointment: ocular pharmacokinetics in rabbits," Journal of Ocular Pharmacology and Therapeutics, vol. 20, no. 2, pp. 169-177, 2004.

[45] C. Giannavola, C. Bucolo, A. Maltese et al., "Influence of preparation conditions on acyclovir-loaded poly- $d$, $l$-lactic acid nanospheres and effect of PEG coating on ocular drug bioavailability," Pharmaceutical Research, vol. 20, no. 4, pp. 584-590, 2003.

[46] M. Fresta, G. Fontana, C. Bucolo, G. Cavallaro, G. Giammona, and G. Puglisi, "Ocular tolerability and in vivo bioavailability of poly(ethylene glycol) (PEG)-coated polyethyl-2-cyanoacrylate nanosphere-encapsulated acyclovir," Journal of Pharmaceutical Sciences, vol. 90, no. 3, pp. 288-297, 2001.

[47] A. Leonardi, C. Bucolo, G. L. Romano et al., "Influence of different surfactants on the technological properties and in vivo ocular tolerability of lipid nanoparticles," International Journal of Pharmaceutics, vol. 470, no. 1-2, pp. 133-140, 2014.

[48] R. Pignatello, C. Bucolo, and G. Puglisi, "Ocular tolerability of Eudragit RS100 ${ }^{\circledR}$ and $\mathrm{RL}_{100}{ }^{\circledR}$ nanosuspensions as carriers for ophthalmic controlled drug delivery," Journal of Pharmaceutical Sciences, vol. 91, no. 12, pp. 2636-2641, 2002. 
[49] C. Bucolo, A. Maltese, F. Maugeri, B. Busà, G. Puglisi, and R. Pignatello, "Eudragit RL100 nanoparticle system for the ophthalmic delivery of cloricromene," The Journal of Pharmacy and Pharmacology, vol. 56, no. 7, pp. 841-846, 2004.

[50] S. Misra, K. Chopra, V. R. Sinha, and B. Medhi, "Galantamineloaded solid-lipid nanoparticles for enhanced brain delivery: preparation, characterization, in vitro and in vivo evaluations," Drug Delivery, vol. 23, no. 4, pp. 1434-1443, 2016.

[51] J. Albuquerque, C. C. Moura, B. Sarmento, and S. Reis, "Solid lipid nanoparticles: a potential multifunctional approach towards rheumatoid arthritis theranostics," Molecules, vol. 20, no. 6, pp. 11103-11118, 2015.

[52] A. Seyfoddin and R. Al-Kassas, "Development of solid lipid nanoparticles and nanostructured lipid carriers for improving ocular delivery of acyclovir," Drug Development and Industrial Pharmacy, vol. 39, no. 4, pp. 508-519, 2013.

[53] A. Seyfoddin, T. Sherwin, D. V. Patel et al., "Ex vivo and in vivo evaluation of chitosan coated nanostructured lipid carriers for ocular delivery of acyclovir," Current Drug Delivery, In press. 

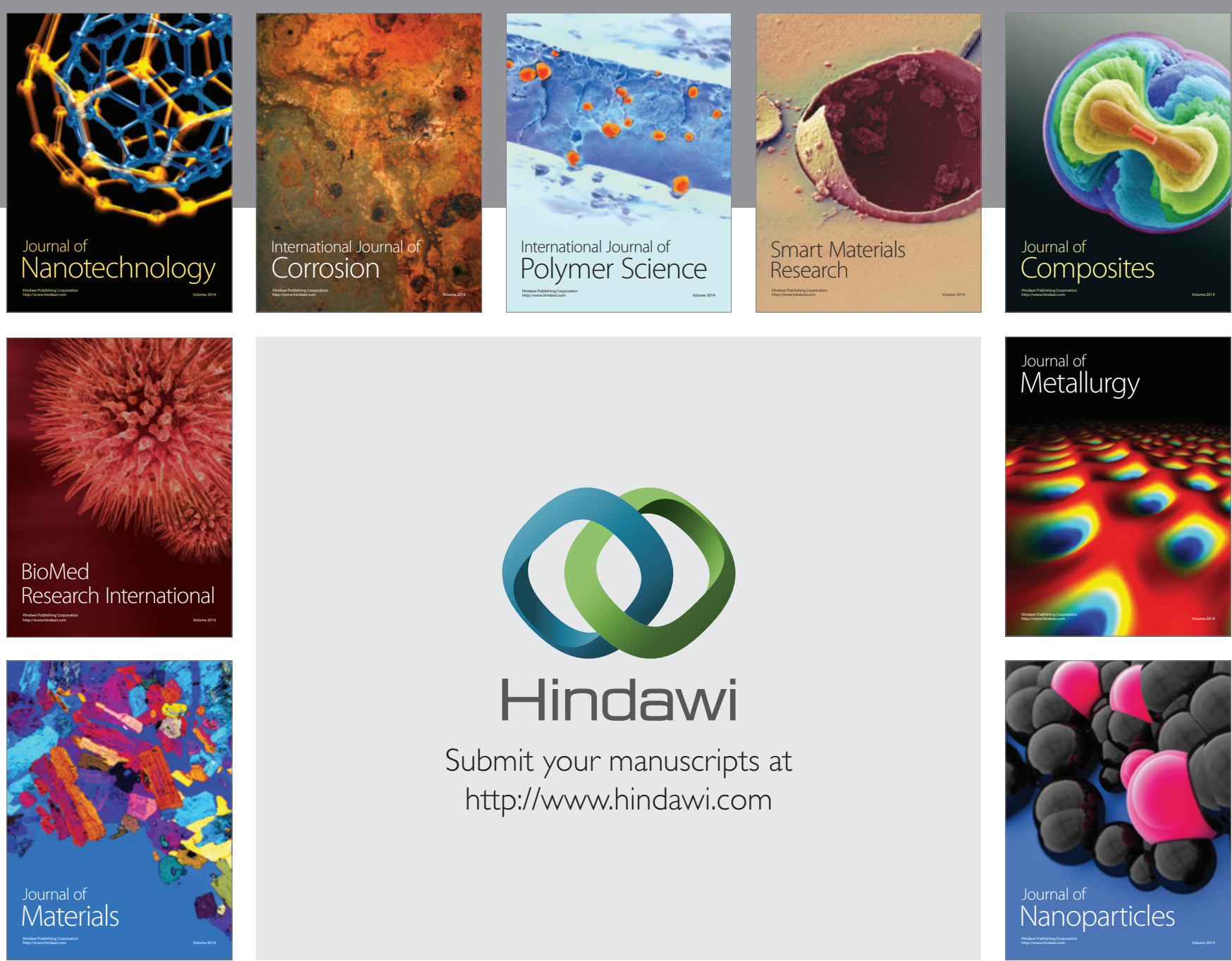

\section{Hindawi}

Submit your manuscripts at

http://www.hindawi.com

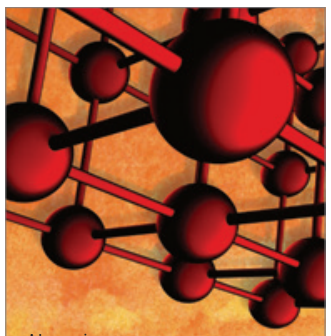

Materials Science and Engineering
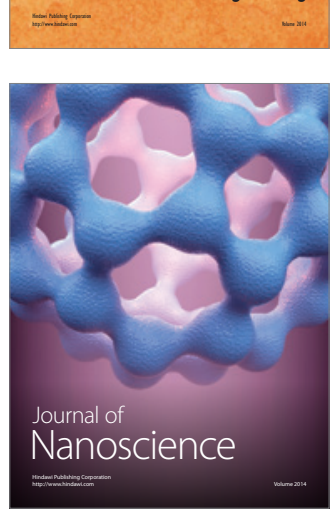
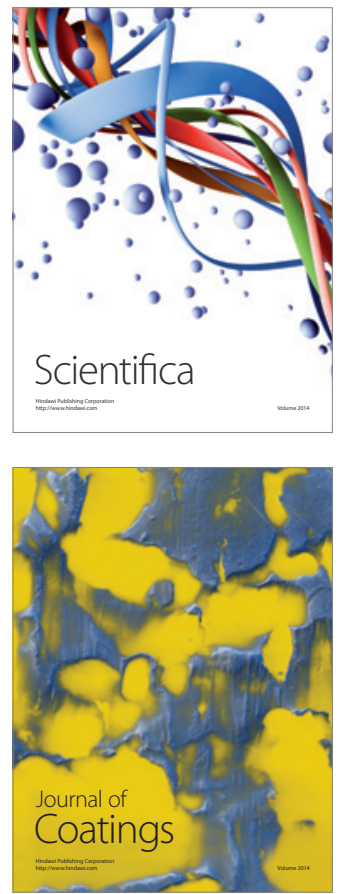
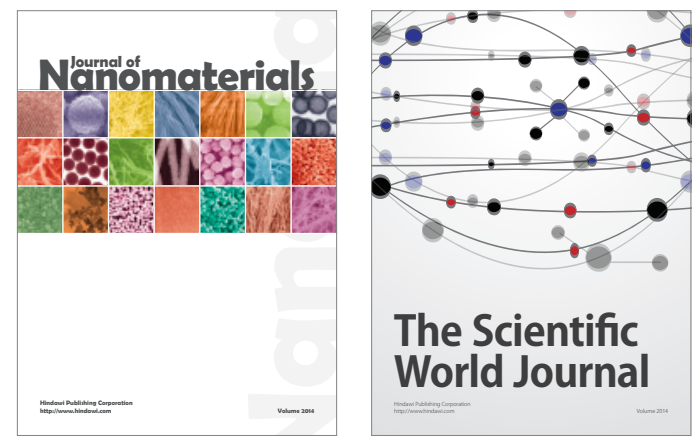

The Scientific World Journal
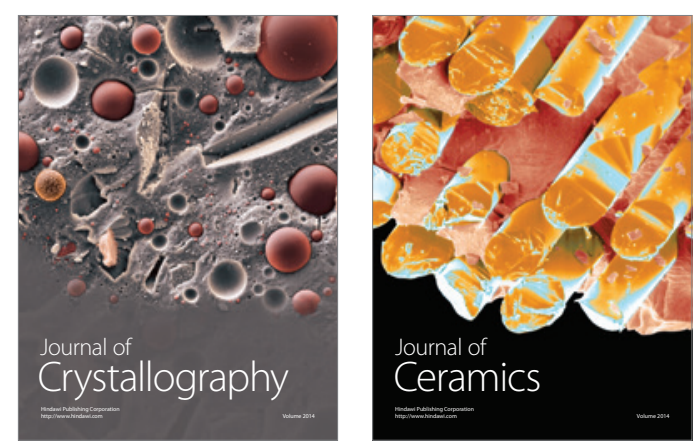
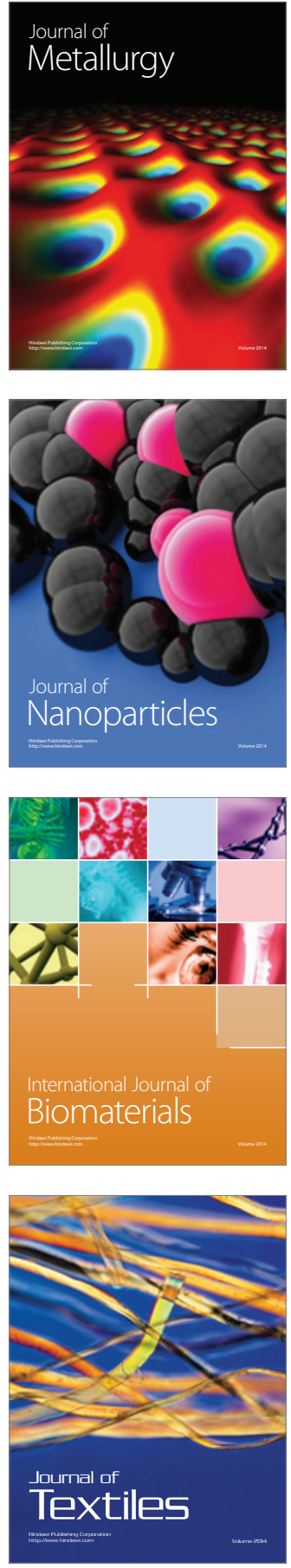